\title{
ELECTRONIC SPECTRA AND TRIPLET STATE PROPERTIES OF SUPERPHANE
}

\author{
D. SCHWEITZER, K.H. HAUSSER, H. VOGLER, H.A. STAAB \\ Max-Planck-Institut, Abteilung Molekulare Physik and Abteilung Organische Chemie, \\ Jahn Strasse 29, 6900 Heidelberg, FRG
}

and

\author{
V. BOEKELHEIDE \\ Department of Chemistry, University of Oregon, \\ Eugene, OR 97403, USA
}

Received 23 June 1986

\begin{abstract}
The emission and the zero-field splitting parameters $D$ and $E$ of the superphane 1 and the $[2,3(1,2,3,4,5)$-cyclophane 2 are investigated. The large number of six and five methylene bridges, respectively, leads to a more rigid molecular frame and to a smaller transanular distance as compared to other [2.2]cyclophanes. The experimental results exhibit characteristic features which are due to these unusual properties; they are discussed and compared with semi-empirical w-electron calculations.
\end{abstract}

\section{Introduction}

The strength of the $\pi$-electron interaction in cyclophanes with identical subunits is determined by the distance as well as by the relative orientation of the two aromatic molecular subunits with respect to each other. It is studied in general by various spectroscopic methods, such as absorption, emission and Raman spectroscopy, and in particular the investigation of the triplet zero-field splitting parameters by magnetic resonance and optically detected magnetic resonance (ODMR). The information obtained concerns the influence of the $\pi$-electron interaction in the lowest excited electronic singlet and triplet state of such cyclophanes on the parameters mentioned above as compared to the corresponding monomer units.

In the case of weak transanular interactions, for instance in [ $n . n]$ paracyclophanes with $n \geqslant 4$, and hence with a large average distance between the two aromatic subunits, the lowest excited electronic singlet and triplet state may be described to a very good approximation by wavefunctions which contain exciton terms only [1-4],

${ }^{n} \psi_{i}^{\mathrm{E}}( \pm)=2^{-1 / 2}\left({ }^{n} \psi_{i}^{\mathrm{A} 1} \psi_{0}^{\mathrm{B}} \pm{ }^{1} \psi_{0}^{\mathrm{An}} \psi_{i}^{\mathrm{B}}\right)$

Here $A$ and $B$ refer to the two molecular units forming the phane, $n=1$ (3) stands for singlet (triplet) state, $i=x^{*}, y^{*}, z^{*}$ designates the spin sublevels in the average principle axis system in case of the triplet state. ${ }^{1} \psi_{0}^{\mathrm{A}}, \mathrm{B}$ and ${ }^{n} \psi_{i}^{\mathrm{A}}, \mathrm{B}$ refer to the complete ground states and excited states localized on the molecular subunits A or B.

With decreasing transanular distance symmetric charge transfer (CT) terms must be taken into account in the total wavefunction [5-8],

${ }^{n} \psi_{i}^{\mathrm{CT}}( \pm)=2^{-1 / 2}\left({ }^{n}\left[\psi_{\mathrm{A}}^{+} \psi_{\mathrm{B}}^{-}\right] \pm^{n}\left[\psi_{\mathrm{A}}^{-} \psi_{\mathrm{B}}^{+}\right]\right)_{i}$

The function ${ }^{n}\left[\psi_{A}^{+} \psi_{B}^{-}\right]$describes the excitation of one electron from $A$ to $B$, for details see refs. [5-9].

Even in the case of the [2.2]paracyclophane, a representative of the $\left[2_{n}\right]$ cyclophanes, in which the two conjugated subunits are situated in a face-toface arrangement, charge transfer terms cannot be 
neglected [10-13] although the excimer excitation following (1) remains predominant.

In addition to the transanular interaction between the $\pi$-systems of the two aromatic six-rings in cyclophane, the through-bond interaction via the connecting methylene bridges is also important for the electronic structure of the ground and excited states [12,14-16]. Only the combined consideration of transanular interaction and through-bond interaction accounts satisfactorily for the observed ionization potentials [14] and for the longest wavelength absorption in the UV spectra in the series of $\left[2_{n}\right]$ cyclophanes [16].

The superphane $1[17]$ and the $\left[2_{5}\right](1,2,3,4,5)-$ cyclophane 2 [18] (see fig. 1) differ in several respects considerably from all other [2.2]cyclophanes which renders the investigation of these phanes particularly interesting. The main differences are:

(i) Both phanes 1 and 2 show with $262.4 \mathrm{pm}$ for 1 [17] and with $270 \mathrm{pm}$ (mean value) for 2 [18] the smallest transanular distance which can be realized in a hydrocarbon phane. This should result in a stronger transanular $\pi$-electron interaction and in a greater importance of the symmetric charge transfer terms.

(ii) The higher number of five and six methylene bridges, respectively, as compared to two methylene bridges in the case of [2.2]paracyclophane 3 is expected to have two consequences: firstly, the through-bond interaction should play a more important role. Secondly, the much more rigid molecular frame of 1 and 2 should result in a much weaker electron-phonon coupling (EPC). Since the EPC is responsible for the unstructured broad band emission spectra $[12,13]$, we expect for 1 and 2 a better resolution of the vibronic structure.
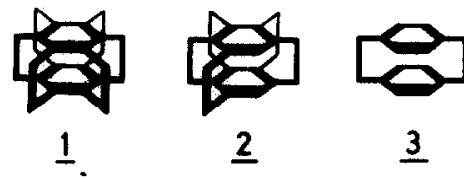

Fig. 1. Molecules: 1 superphane $\left[2{ }_{6}\right](1,2,3,4,5,6)$-cyclophane; 2 $[2,1(1,2,3,4,5)$-cyclophane; 3 [2.2]paracyclophane. (iii) The superphane 1 possesses a sixfold symmetry axis and hence the HOMO and LUMO of both subunits are degenerate [14]. Consequently the approximation made for eq. (2) is not sufficient but first-order configuration interaction [19] is expected to be of crucial importance.

\section{Experimental}

ODMR transitions and emission spectra at 1.3 $\mathrm{K}$ were observed from polycrystalline samples of purified superphane $1 \quad([26](1,2,3,4,5,6)$-cyclophane) and $[2],(1,2,3,4,5)$-cyclophane 2 as well as of samples of 1 and 2 dissolved in low concentration $\left(\leqslant 10^{-3} \mathrm{~mol}\right)$ in methylcyclohexane $(\mathrm{MCH})$.

The samples were placed into a liquid helium cryostat and the temperature reduced to $1.3 \mathrm{~K}$ by pumping. Optical excitation at $313 \mathrm{~nm}$ was performed with a HBO 100 mercury lamp in combination with a $0.25 \mathrm{~m}$ monochromator (Shoeffel GM 250) and additional filters (Schott UV-R-310 + UG 11).

The fluorescence and phosphorescence was monitored at right angles to the excitation path with a $0.85 \mathrm{~m}$ double monochromator (Spex 1402) with holographic gratings and spatial filtering option equipped with a cooled $\left(-50^{\circ} \mathrm{C}\right)$ photomultiplier (RCA 31034A02) in combination with a photon counting system (PAR 1109).

The ODMR apparatus was essentially the same as described earlier [20]. Signal averaging was achieved by using a transient recorder (Biomation 8100 ) interfaced to a computer system (Dietz 621, $48 \mathrm{~K}$ ). Tunable band-pass filters or a combination of low- and high-pass filters were used to reduce the harmonic and spurious frequencies of the microwave sweepers (HP 8620A) by at least $60 \mathrm{~dB}$.

A Raman spectrum of a polycrystalline sample of 1 was recorded at $1.3 \mathrm{~K}$ by using an argon ion laser (Coherent CR6UV) with an additional laser monochromator and an interference filter (Schott $514 \mathrm{~nm}$ ) as excitation source. A power of $50 \mathrm{~mW}$ at $514 \mathrm{~nm}$ focused on the sample was sufficient in order to obtain good quality spectra. Raman lines could be recorded for frequencies larger than 10 $\mathrm{cm}^{-1}$. 


\section{Results}

Figs. 2 and 3 show the emission spectra of 1 and 2 in low concentration ( $\leqslant 10^{-3} \mathrm{~mol}$ ) in $\mathrm{MCH}$ as well as of neat polycrystalline samples at $1.3 \mathrm{~K}$. In addition, fig. 4 shows the fluorescence and phosphorescence of the polycrystalline sample of 1 under higher resolution, and fig. 5 the lower-energetic part of the Raman spectrum of the same sample at $1.3 \mathrm{~K}$ which was not investigated in ref. [21].
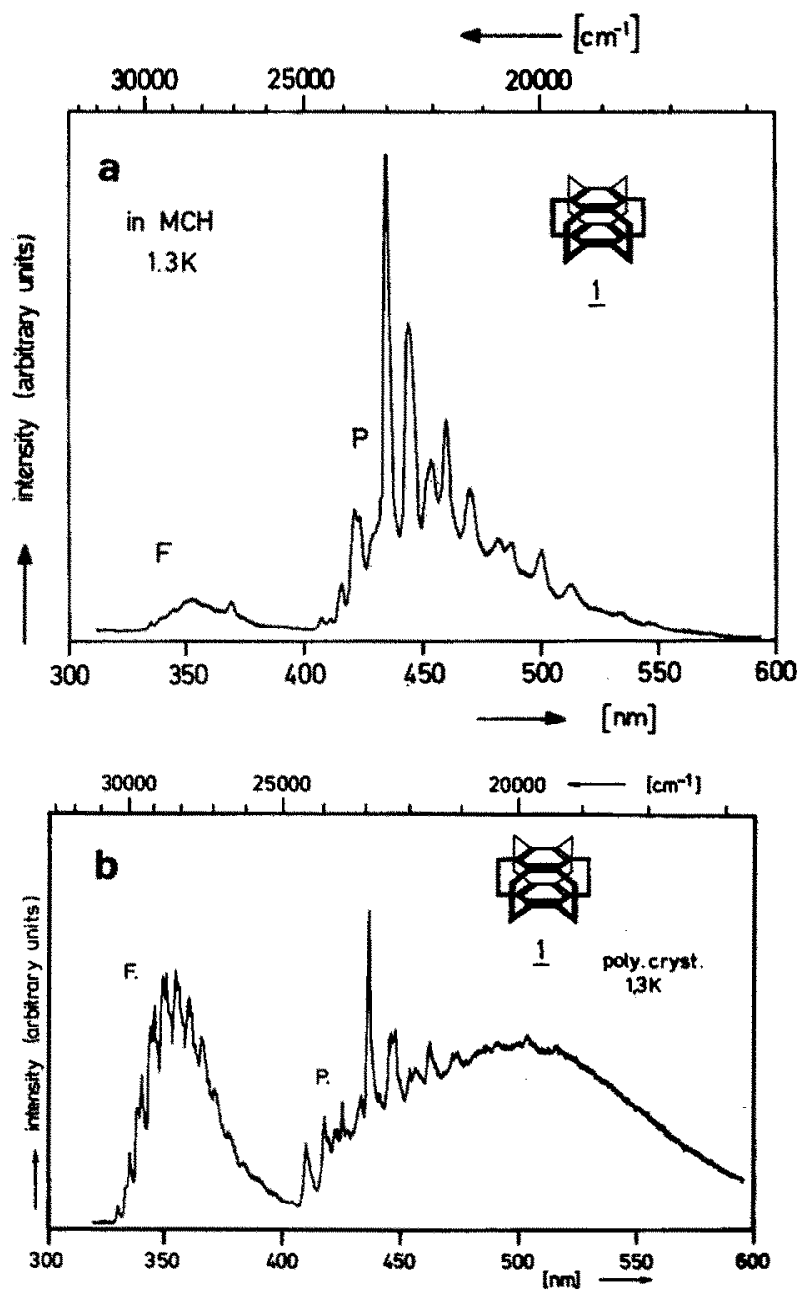

Fig. 2. Fluorescence (F) and phosphorescence (P) of superphane 1 at $1.3 \mathrm{~K}$. (a) In low concentration $\left(<10^{-3} \mathrm{~mol}\right)$ in methylcyclohexane (MCH); (b) of a neat polycrystalline sample.

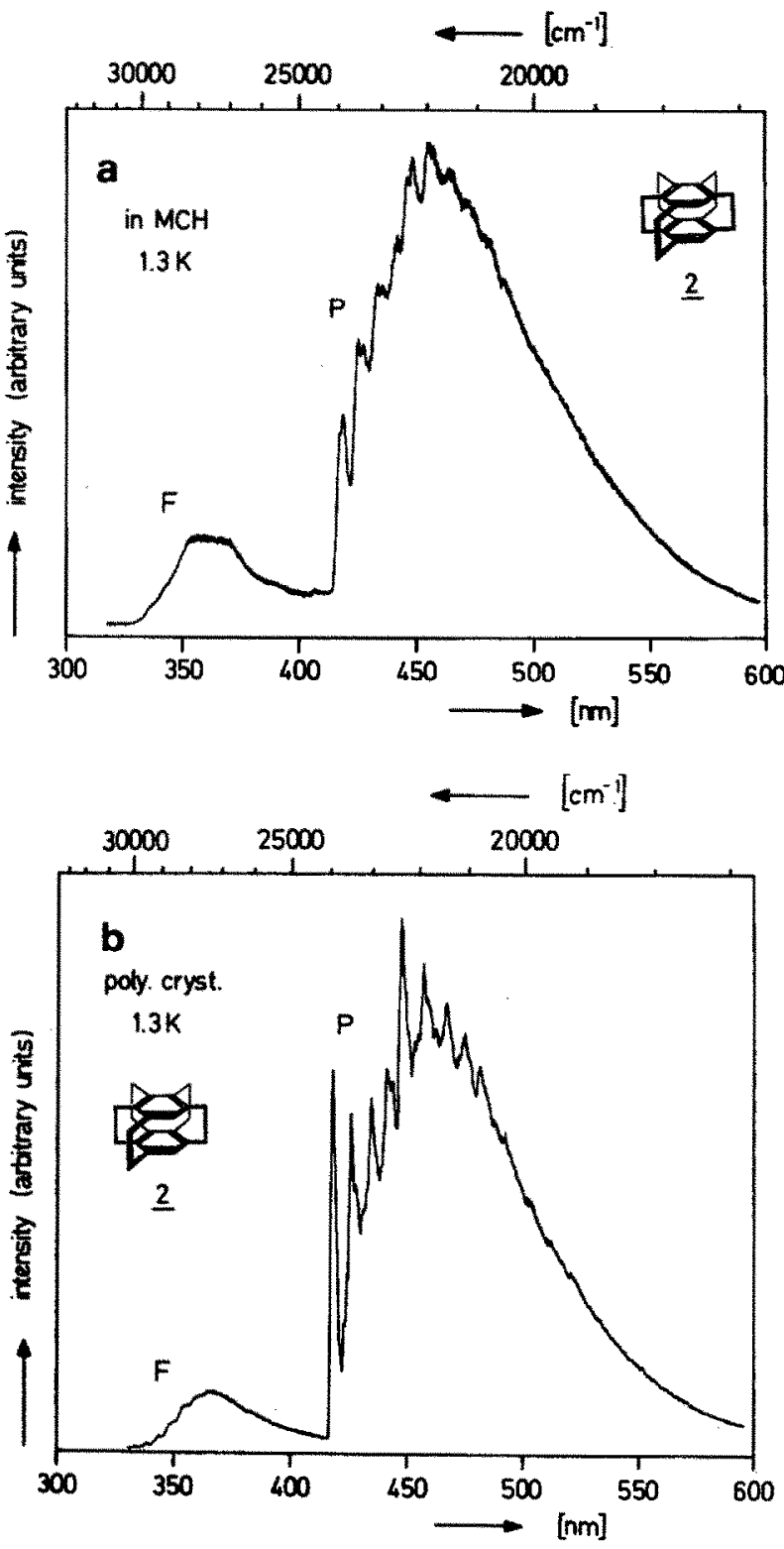

Fig. 3. Fluorescence (F) and phosphorescence (P) of $[2,5]$ $(1,2,3,4,5)$-cyclophane 2 at $1.3 \mathrm{~K}$. (a) In low concentration $\left(<10^{-3} \mathrm{~mol}\right)$ in methylcyclohexane $(\mathrm{MCH}) ;(b)$ of a neat polycrystalline sample.

Table 1 contains the 0,0-bands of the fluorescence and phosphorescence of the polycrystalline samples of 1 and 2 and the phosphorescence of both in low concentration in $\mathrm{MCH}$. In table 2 the vibronic bands observed in the fluorescence 

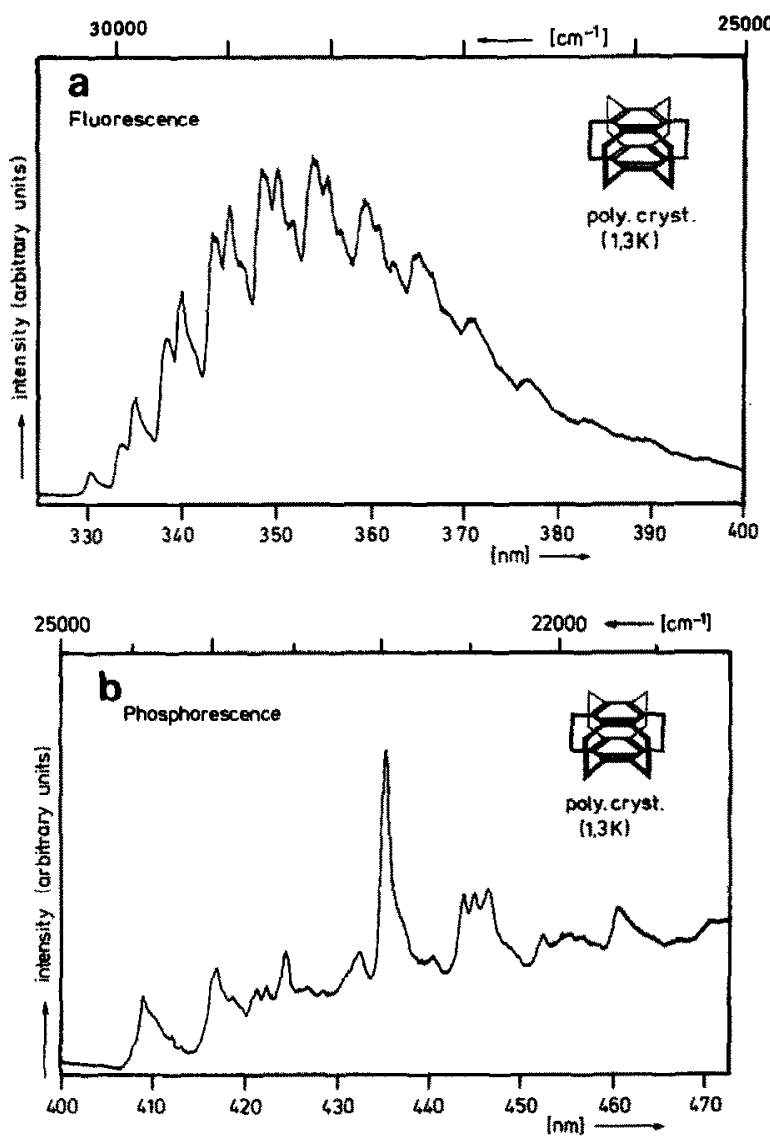

Fig. 4. (a) Fluorescence and (b) phosphorescence of superphane 1 in a polycrystalline sample at $1.3 \mathrm{~K}$ under higher resolution with respect to fig. 2 .

and phosphorescence of the polycrystalline sample of 1 are compiled as well as the lower-energetic Raman lines of fig. 5. The triplet zero-field splitting parameters $D$ and $E$. of 1 and 2 in low concentration in $\mathrm{MCH}$ as well as those of neat polycrystalline samples as obtained by ODMR in

Table 1

0,0 -bands in $\mathrm{cm}^{-1}$ of the fluorescence and phosphorescence of neat polycrystalline samples of 1 and 2 and of the phosphorescence of 1 and 2 in low concentration in $\mathrm{MCH}$

\begin{tabular}{lcclll}
\hline Sample & \multicolumn{2}{l}{ Fluorescence } & & \multicolumn{2}{l}{ Phosphorescence } \\
\cline { 2 - 3 } \cline { 5 - 6 } & 1 & 2 & & 1 & 2 \\
\hline crystalline & 30271 & 29911 & & 24451 & 24051 \\
in MCH & - & - & & 24572 & 24038 \\
\hline
\end{tabular}

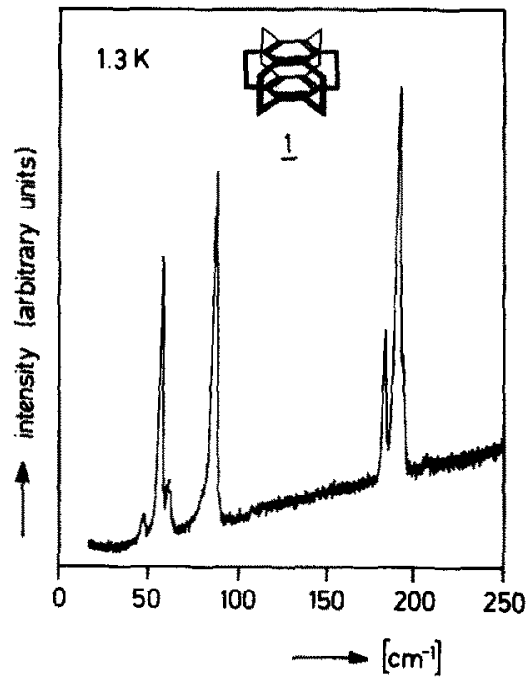

Fig. 5. Low-energetic part of the Raman spectrum of a polycrystalline sample of superphane 1 at $1.3 \mathrm{~K}$.

Table 2

Vibronic bands observed in the fluorescence and phosphorescence as well as Raman lines of the low-energetic part of the Raman-spectrum of a neat polycrystalline sample of 1 . ( $(w=$ very weak, $w=$ weak, $s=$ strong, $v$ wery strong)

\begin{tabular}{lll}
\hline $\begin{array}{l}\text { Fluorescence } \\
\left(\mathrm{cm}^{-1}\right)\end{array}$ & $\begin{array}{l}\text { Phosphorescence } \\
\left(\mathrm{cm}^{-1}\right)\end{array}$ & $\begin{array}{l}\text { Raman }^{\text {b) }} \\
\left(\mathrm{cm}^{-1}\right)\end{array}$ \\
\hline & & $51 \mathrm{w}$ \\
$59 \mathrm{~s}$ \\
& & $65 \mathrm{w}$ \\
112 & 123 & $89 \mathrm{~s}$ \\
& & $185 \mathrm{w}-\mathrm{s}$ \\
& & $192 \mathrm{~s}$ \\
& & $210 \mathrm{vw}$ \\
298 & 240 & \\
& 319 & $371 \mathrm{w}$ \\
426 & & $379 \mathrm{ww}$ \\
& 435 & $444 \mathrm{w}-\mathrm{s}$ \\
& & $453 \mathrm{w}-\mathrm{s}$ \\
& & $470 \mathrm{vw}$ \\
& & $581 \mathrm{vs}$ \\
& $637 \mathrm{w}-\mathrm{s}$ \\
& & $682 \mathrm{~s}$ \\
& & $\vdots$ \\
& & $1480 \mathrm{vw}$ \\
\hline
\end{tabular}

a) Error $\pm 15 \mathrm{~cm}^{-1}$.

b) Error $\pm 2 \mathrm{~cm}^{-1}$. 
Table 3

Triplet zero-field splitting parameters $|D|$ and $|E|$ (in $\mathrm{cm}^{-1}$ ) of 1 and 2 in low concentration $\left(\leqslant 10^{-3}\right.$ mol) in methylcyclohexane $(\mathrm{MCH})$ as well as of neat polycrystalline samples as obtained by ODMR in zero field at $1.3 \mathrm{~K}$. For the crystalline sample of 2 two different $\mathrm{X}$-traps could be observed. The $D$ and $E$ values of the monomer hexamethylbenzene are not included for comparison because it is not planar

\begin{tabular}{|c|c|c|c|c|}
\hline \multirow[t]{2}{*}{ Sample } & \multicolumn{2}{|l|}{$|D|\left(\mathrm{cm}^{-1}\right)$} & \multicolumn{2}{|l|}{$|E|\left(\mathrm{cm}^{-1}\right)$} \\
\hline & 1 & 2 & 1 & 2 \\
\hline $\begin{array}{l}\text { in MCH } \\
\text { polycrystalline }\end{array}$ & $\begin{array}{l}0.1072( \pm 2) \\
0.1026( \pm 1)\end{array}$ & $\begin{array}{l}0.0850( \pm 2) \\
0.0909( \pm 1) X_{1} \\
0.0884( \pm 1) X_{2}\end{array}$ & $\begin{array}{l}0.0063( \pm 2) \\
0.0101( \pm 1)\end{array}$ & $\begin{array}{l}0.0136( \pm 2) \\
0.0187( \pm 1) X_{1} \\
0.0186( \pm 1) X_{2}\end{array}$ \\
\hline
\end{tabular}

zero field at $1.3 \mathrm{~K}$ are compiled in table 3 . In the case of the crystalline sample of 2 two very similar sets of zero-field splitting parameters are given due to the fact that two different $X$-traps could be observed and therefore six ODMR transitions were found.

\section{Discussion}

The fluorescence and phosphorescence spectra of the polycrystalline samples of 1 and 2 (fig. 2) show clearly detectable 0,0-bands (table 1) and resolved vibronic structure (table 2 ), while for 1 and 2 in low concentration in $\mathrm{MCH}$ (fig. 2) the relatively weak fluorescence is broad band and more or less structureless while the more intense phosphorescence shows again vibronic structure. The detectable 0,0-bands and the resolved vibronic structures are in contrast to the usually observed broad band and structureless emissions of [2.2]and [3.3]paracyclophane [12], but can be explained by a much weaker electron-phonon coupling (EPC) for 1 and 2 due to the much more rigid frame caused by the six respectively five methylene bridges.

While in [2.2] and [3.3]paracyclophane the strong coupling of several low-frequency dimer modes to the electronic transitions (strong EPC) with coupling strengths $S=11-13$ [13] leads to a relatively large equilibrium shift of the nuclear positions under electronic excitation and therefore to the structureless and broad band emissions [12,13], here in 1 and 2 the EPC coupling strength is much weaker ( $S \approx 1$ for the $1480 \mathrm{~cm}^{-1}$ mode and $S=2-3$ for the other observed modes; see table 2 and ref. [13] for the definition of the coupling strength $S$ ). This weaker EPC results in the resolved vibronic structure.

At the moment none of the observed lowfrequency modes of 1 (table 2) can be identified as a special dimer mode (for instance breathing or torsional mode) - as it was possible in the case of [2.2]paracyclophane 3 [13]. This is due to the fact that superphane (1) has $D_{6 h}$ symmetry in the ground state, and therefore neither $A_{1 g}$ nor $A_{1 u}$ modes are IR or Raman active. Hence it is not surprising that none of the low-energetic bands of 1 observed in the fluorescence or phosphorescence emissions is found in the Raman spectrum in fig. 5 as can be seen in table 2 . Only the $0,0-1480$ $\mathrm{cm}^{-1}$ band can be clearly seen in the emission spectra as well as in the Raman spectrum and belongs to a typical $\mathrm{C}-\mathrm{C}$ ring vibration. The larger intensity of this band in the phosphorescence with respect to the 0,0-band is due to the fact that in an ideal $D_{6 h}$ symmetry the 0,0-band should be symmetry forbidden, but because the phosphorescence in the polycrystalline material originates from $\mathrm{X}$ traps, this ideal symmetry is probably not preserved for the molecules forming the traps.

The fluorescence and phosphorescence 0,0 bands of 1 in a crystalline sample are shifted to the red by 93 and $151 \mathrm{~cm}^{-1}$ with respect to the corresponding 0,0-bands of [2.2]paracyclophane (3) [13]. This can be qualitatively understood as originating from the stronger transanular interaction (TAI) and the equally stronger through-bond interaction (TBI) in the case of 1 although an even larger red shift might have been expected.

On the other hand, the results of the triplet zero-field splitting parameters $D$ and $E$ depicted 
in table 3 are rather surprising. $D$ is given by

$D=\left(C_{1 i}^{(j)}\right)^{2} D_{\mathrm{E}}+\left(C_{2 i}^{(j)}\right) D_{\mathrm{CT}}$

where $\left(C_{1 i}\right)^{2}+\left(C_{2 i}\right)^{2}=1$. $D_{\mathrm{E}}$ originates from the exciton term (1) and $D_{\mathrm{Cr}}$ from the CT term (2). The CT contribution $D_{\mathrm{CT}}$ is negative if the $z$-axis coincides with the axis perpendicular to the plane and through the center of the six rings and if $z$ defines the operator associated with $D$. Since this is true for all phanes discussed here, the existence of $\mathrm{CT}$ terms in the wavefunction results always in a reduction of $D$ as compared to the monomer $D_{\mathrm{M}}$, the value of the isolated subunit $(\mathrm{M}=$ monomer) $[10,22]$. Because of the stronger transanular interaction (TAI) and the stronger through-bond interaction (TBI) one would expect a stronger contribution of the CT terms to the wavefunction and hence a considerably lower $D$ value for 1 as compared to 3 . However, the $D$ value of 1 in $\mathrm{MCH}$ is nearly identical with the $D$ value of the second set observed for 3 in $\mathrm{MCH}$ [12]. The fact that the $D$ value of 2 is somewhat smaller in comparison to 1 and 3 can be understood because of a marked out-of-plane deformation in 2 at the carbon positions in the ring which are not connected by $-\mathrm{CH}_{2}-\mathrm{CH}_{2}-$ groups. We shall therefore restrict the discussion in the following part to the molecules 1 and 3.

In order to understand the unexpected results discussed above we have calculated the contributions of the TAI and the through-bond interactions (TBI) in 1 and 3.

Superphane 1 exhibits the smallest transanular distance in a hydrocarbon phane which leads necessarily to large TAI. Consequently, it is doubtful whether the electronic structure of the excited states of 1 can be adequately described by the wavefunctions of eqs. (1) and (2) or whether an ansatz for the whole phane (super molecule ansatz) has to be used. But even with a super molecule ansatz the wavefunctions can be represented by eqs. (1) and (2) if their terms are interpreted differently. (i) The charge transfer term (2) includes all one-electron excitations from orbitals in $A$ into those in $B$ and vice versa. Herewith we assume that higher excitations are unimportant within our semi-empirical approach. (ii) ${ }^{n} \psi_{i}^{\mathrm{A}} \mathrm{de}-$ scribes excitations within the real subunit $A$ which is characterized by TAI and TBI between A and B. Thus ${ }^{n} \psi_{i}^{\mathrm{A}}$ is no longer interpreted as an excited state of the isolated subunit $A$ which would be identical to that of the monomer within the $\pi$-electron approximation.

Since ab initio procedures are hardly feasible for the study of excited triplet states of such extended systems as cyclophanes, we used a semiempirical $\pi$-electronic approach by Spanget-Larsen [16] which guarantees a balanced account of TAI and TBI in a cyclophane. In order to simplify the calculations we replaced the two pseudomethylene $\pi$-orbitals which describe the $-\left(\mathrm{CH}_{2}\right)_{2}-$ bridge bond orbitals by a single $\sigma$-orbital with a distance of $152 \mathrm{pm}$ from both benzene rings in the phane. Consequently, the out-of-phase combination of the pseudomethylene orbitals needs no longer be annihilated. This replacement has no recognizable effect on the calculated values since the in-phase combination of the pseudomethylene orbitals has $\sigma$-type nature. The excited triplet states are obtained by configuration interaction between all singly excited configurations. The ZFS parameter $D$ is calculated as described in ref. [10] with a reduction of the ortho $D_{s t}$ integral by $10.3 \%$ so that the calculated and the experimental $D$ values of benzene agree. The calculations are performed by using idealized geometries with coplanar benzene rings with a separation of $299 \mathrm{pm}$ for 3 (mean value [23] of all transanular distances between carbon atoms which are directly one upon another) and $262 \mathrm{pm}$ for 1 [24]. All calculated values are given in table 4 .

Within the magnetic axes system the $D$ parameter for the triplet state can be calculated as an expectation value $D=\left\langle{ }^{3} \phi^{(j)}|\hat{D}|^{3} \phi^{(j)}\right\rangle$ of an operator $\hat{D} \cdot{ }^{3} \phi^{(j)}$ denotes the antisymmetric function of the spatial coordinates for the two unpaired electrons. This function reads as

${ }^{3} \phi^{(j)}=C_{1}^{(j) 3} \psi^{\mathrm{E}}( \pm)+C_{2}^{(j) 3} \psi^{\mathrm{CT}}( \pm)$

with

${ }^{3} \psi^{\mathrm{E}}( \pm)=2^{-1 / 2}\left({ }^{3} \psi^{\mathrm{A}} \pm{ }^{3} \psi^{\mathrm{B}}\right)$,

${ }^{3} \psi^{\mathrm{CT}}( \pm)=2^{-1 / 2}\left({ }^{3}\left[\psi_{\mathrm{A}}^{+} \psi_{\mathrm{B}}^{-}\right] \pm^{3}\left[\psi_{\mathrm{A}}^{-} \psi_{\mathrm{B}}^{+}\right]\right)$.

Assuming that only singly excited configurations 
Table 4

Calculated and experimental (in parentheses) values of the triplet excitation energies $T_{1}$ and $T_{2}$ (in eV) as well as the $D$ values (in $\mathrm{cm}^{-1}$ ) and the parameters $x$ and $y$ as used in eq. (10) for 1 and 3

\begin{tabular}{|c|c|c|c|c|c|c|c|c|c|}
\hline & $T_{1}$ & $T_{2}$ & $D_{\mathrm{A}}$ & $D_{\mathrm{AB}}$ & $x$ & $y$ & $2 x D_{\mathrm{A}}$ & $\mathrm{y} D_{\mathrm{AB}}$ & $D$ \\
\hline 1 & $2.09(3.03)$ & 2.35 & 0.066 & -0.076 & 1.00 & 0.32 & 0.131 & -0.024 & $\begin{array}{c}0.107 \\
(0.1072\end{array}$ \\
\hline 3 & $2.14(3.05)$ & 2.43 & 0.069 & -0.033 & 0.89 & 0.50 & 0.123 & -0.016 & $\begin{array}{c}0.107 \\
(0.1073)\end{array}$ \\
\hline \multicolumn{10}{|c|}{ without through-bond interaction } \\
\hline 1 & 1.35 & - & 0.061 & -0.097 & 1.00 & 0.32 & 0.122 & -0.031 & 0.091 \\
\hline 3 & 1.56 & - & 0.070 & -0.036 & 0.89 & 0.50 & 0.125 & -0.018 & 0.107 \\
\hline \multicolumn{10}{|c|}{ without transanular interaction } \\
\hline 1 & 1.99 & - & 0.062 & -0.075 & 1.00 & 0.32 & 0.124 & -0.024 & 0.100 \\
\hline 3 & 2.32 & - & 0.073 & -0.027 & 0.89 & 0.50 & 0.130 & -0.014 & 0.116 \\
\hline
\end{tabular}

are taken into account in (5) and (6), we arrive at formula (3) with

$$
\begin{aligned}
D_{\mathrm{E}}= & \frac{1}{2}\left(\left\langle^{3} \psi^{\mathrm{A}}|\hat{D}|^{3} \psi^{\mathrm{A}}\right\rangle+\left\langle{ }^{3} \psi^{\mathrm{B}}|\hat{D}|^{3} \psi^{\mathrm{B}}\right\rangle\right. \\
& \left. \pm 2\left\langle{ }^{3} \psi^{\mathrm{A}}|\hat{D}|^{3} \psi^{\mathrm{B}}\right\rangle\right) \\
D_{\mathrm{CT}}= & \left\langle{ }^{3} \psi^{\mathrm{CT}}( \pm)|\hat{D}|^{3} \psi^{\mathrm{CT}}( \pm)\right\rangle .
\end{aligned}
$$

Another grouping of terms is also possible leading to

$D=2 D_{\mathrm{A}}+D_{\mathrm{AB}}$,

with

$$
\begin{aligned}
D_{\mathrm{A}}= & \frac{1}{2}\left(C_{1}^{(j)}\right)^{2}\left\langle{ }^{3} \psi^{\mathrm{A}}|\hat{D}|^{3} \psi^{\mathrm{A}}\right\rangle \\
= & \frac{1}{2}\left(C_{1}^{(j)}\right)^{2}\left\langle{ }^{3} \psi^{\mathrm{B}}|\hat{D}|^{3} \psi^{\mathrm{B}}\right\rangle=D_{\mathrm{B}}, \\
D_{\mathrm{AB}}= & \pm\left(C_{1}^{(j)}\right)^{2}\left\langle{ }^{3} \psi^{\mathrm{A}}|\hat{D}|^{3} \psi^{\mathrm{B}}\right\rangle \\
& +\left(C_{2}^{(j)}\right)^{2}\left\langle{ }^{3} \psi^{\mathrm{CT}}( \pm)|\hat{D}|^{3} \psi^{\mathrm{CT}}( \pm)\right\rangle .
\end{aligned}
$$

Here $D_{\mathrm{A}}\left(D_{\mathrm{B}}\right)$ refers to a contribution from subunit $A(B)$ alone, whereas $D_{A B}$ refers to both subunits.

Without TAI and TBI no CT terms are present in the triplet wavefunction and eq. (3) reduces to $D=D_{\mathrm{E}}$. However, eqs. (7) and (8) reveal that $D$ then still contains the intersubunit term $D_{\mathrm{AB}}=$ $\pm\left\langle{ }^{3} \psi^{\mathrm{A}}|\hat{D}|^{3} \psi^{\mathrm{B}}\right\rangle$ due to the two-electron nature of the operator $\hat{D}$ [10]. In case of non-vanishing TAI and TBI a unique statement of the CT character with respect to ZFS parameters is no longer possible due to the occurrence of the intersubunit term.

Within our semi-empirical MO approach we can consider a $\left[2_{n}\right]$ cyclophane to consist of the two equivalent six-ring units $A$ and $B$ and the set of $C$ of all methylene bridges. In analogy to eq. (7) the $D$ parameter can be written as a sum

$D=2 D_{\mathrm{A}}+D_{\mathrm{AB}}+D_{\mathrm{C}}+2 D_{\mathrm{AC}}$,

where $D_{\mathrm{A}}$ is the contribution of the six ring unit $\mathrm{A}$ $\left(D_{\mathrm{A}}=D_{\mathrm{B}}\right), D_{\mathrm{C}}$ that of the methylene bridges, $D_{\mathrm{AB}}$ the transanular contribution with respect to the two six rings $\mathrm{A}$ and $\mathrm{B}$, and $D_{\mathrm{AC}}$ a contribution from the six-ring unit $A$ and the methylene bridges $\left(D_{\mathrm{AC}}=D_{\mathrm{BC}}\right)$.

In case of 3 the calculated transanular term $D_{\mathrm{AB}}$ turned out to be numerically too large [10]. Therefore we replaced $D_{A B}$ by $y D_{A B}$ with $y<1$. For 1 we expect a smaller $y$ parameter since the transanular integrals which have been calculated within a point-dipole model [10] tend to be overestimated with decreasing interplanar separation. The deformations of the benzene rings $A$ and $B$ in $\left[2_{n}\right]$ cyclophanes lead to a reduction of the contribution $D_{\mathrm{A}}=D_{\mathrm{B}}$. This can be taken into account by introducing a "deformation" parameter $x$ and replacing $D_{\mathrm{A}}$ by $x D_{\mathrm{A}}[10]$. Furthermore, our calculations revealed that $D_{\mathrm{C}}$ and $D_{\mathrm{AC}}$ are negligible and neet not to be considered explicitly. Consequently a discussion of $D$ can be based on the equation

$D=2 x D_{\mathrm{A}}+y D_{\mathrm{AB}}$.

With $y=0.5[10]$ for 3 we can determine the deformation parameter $x$ by equating experimental and calculated $D$ value, see table 4 . Similarly 
the parameter $y$ can be obtained in case of 1 since here the benzene rings are not performed [24] (this should be valid also in the triplet state due to the rigidity of 1) so that $x=1$. As expected we find $y(\mathbf{1})<y(3)$. Without consideration of benzene ring deformation we would obtain $D_{\mathrm{A}}(1)<D_{\mathrm{A}}(3)$ and $\left|y D_{\mathrm{AB}}(\mathbf{1})\right|>\left|y D_{\mathrm{AB}}(3)\right|$ due to the smaller interplanar separation and hence the larger TAI in 1 with respect to 3 . Consequently, one would expect $D(1)<D(3)=0.122 \mathrm{~cm}^{-1}$ according to our calculated values. The deformation in the benzene rings in 3 leads to a considerable reduction of $D$ since $D_{\mathrm{A}}(1)>x D_{\mathrm{A}}(3)$. This rationalizes the almost agreeing $D$ values of 1 and 3 .

In table 4 also the $D_{\mathrm{A}}$ and $D_{\mathrm{AB}}$ terms are given which are obtained by neglecting TBI. In agreement with our earlier findings [10] both terms are not influenced significantly by TBI in case of 3 . However, $D_{\mathrm{A}}$ and $D_{\mathrm{AB}}$ of 1 and hence $D$ are smaller without TBI. This demonstrates that due to the maximum number of methylene bridges in 1 TBI is crucial beside TAI not only for the magnitude of ionization potentials [14] and singlet or triplet excitation energies [16], but also for the ZFS parameter $D$.

Our calculation leads to a qualitatively satisfactory interpretation of ZFS parameters for 1 and 3 . It would be interesting to check the reliability of our approach with other related systems with different interplay of TAI, TBI and deformation. Unfortunately, further experimental values are so far not known.

The comparison of $D$ values with and without TAI in table 4 reveals that TAI reduces $D_{\mathrm{A}}$ of 3 but increases $D_{\mathrm{A}}$ and 1 . Since $D_{\mathrm{AB}}<0$ for 1 and 3 and all other $\left[2_{n}\right]$ cyclophanes [25] the term $D_{\mathrm{AB}}$ reduces $D$ already in the absence of TAI and TBI. This shows the similar nature of the lowest triplet state in the series of $[2 n]$ cyclophanes. Obviously TAI causes a reduction of $D$ for 3 but an increase of $D$ for 1 .

A triplet state is characterized by two unpaired electrons which can be both in the same subunit $\mathrm{A}, \mathrm{B}$ or $\mathrm{C}$ of the phane or in different subunits at a certain time. We define $\Gamma_{\mathrm{XY}}$ as the probability to find one of the unpaired electrons in subunit $X$ while the other one is in $Y$, i.e., $\Gamma_{\mathrm{AA}}$ corresponds to the unpaired electron distribution ( $A \uparrow \uparrow B C$ ), whereas $\Gamma_{\mathrm{AB}}$ to (A $\uparrow \mathrm{B} \uparrow \mathrm{C}$ ) etc. Without $\mathrm{TBI}$ and irrespective of any TAI the $\Gamma_{\mathrm{XY}}$ are fixed for all $\left[2_{n}\right]$ cyclophanes with equivalent subunits $A$ and $B$ due to symmetry as follows: $\Gamma_{\mathrm{AA}}=\Gamma_{\mathrm{BB}}=25 \%$, $\Gamma_{\mathrm{AB}}=50 \%, \Gamma_{\mathrm{AC}}=\Gamma_{\mathrm{BC}}=0 \%$, and $\Gamma_{\mathrm{CC}}=0 \%$. The occurrence of a non-vanishing $\Gamma_{\mathrm{AB}}$ is responsible for the transanular contribution $D_{\mathrm{AB}}$. With TAI and TBI the probabilities change to $\Gamma_{\mathrm{AA}}=24.0 \%$, $\Gamma_{\mathrm{AB}}=48.0 \%, \Gamma_{\mathrm{AC}}=2.0 \%, \Gamma_{\mathrm{CC}}=0.0 \%$ for 3 and $\Gamma_{\mathrm{AA}}=22.0 \%, \quad \Gamma_{\mathrm{AB}}=44.1 \%, \quad \Gamma_{\mathrm{AC}}=5.9 \%, \quad \Gamma_{\mathrm{CC}}=$ $0.0 \%$ for 1 . The decrease of $\Gamma_{\mathrm{AA}}$ and $\Gamma_{\mathrm{AB}}$ is connected with an increase of $\Gamma_{\mathrm{AC}}$ due to a probability to find one of the unpaired electrons in the methylene bridges. The probability $\Gamma_{\mathrm{CC}}$ for finding both unpaired electrons in the bridges is negligible. Despite a considerable $\Gamma_{\mathrm{AC}}$ this does not produce a significant $D_{\mathrm{AC}}$ term due to the smallness of the corresponding integrals. Changes in $\Gamma_{\mathrm{AA}}$ or $\Gamma_{\mathrm{AB}}$ should be reflected in alterations of $D_{\mathrm{A}}$ and $D_{\mathrm{AB}}$. However, it should be stressed that $D_{\mathrm{A}}$ and $D_{\mathrm{AB}}$ also depend on details of the electron distribution within the subunits. This is clearly demonstrated if one compares 3 with 1 where only in the former the decrease of $\Gamma_{\mathrm{AA}}$ by TAI is reflected in the reduction of $D_{\mathrm{A}}$.

Our calculated triplet excitation energies $T_{1}$ for 1 and 3 are about $1 \mathrm{eV}$ too low since the parametrization [16] used in our calculations was chosen in order to reproduce singlet excitation energies. Different parameters would be needed for triplet states due to different core correlation energy contributions [26], however, the variations of triplet excitation energies in a series of conjugated hydrocarbons are nevertheless correctly reproduced $[16,25]$.

For 3 the $D$ values of the first and the second triplet state could be observed [12]. This is most likely due to the fact that both states exhibit similar energies so that in some $X$-traps their order is reversed due to a stabilization of the second triplet state by deformations. The small energy splitting between both states is reproduced by our calculations $\left(T_{2}-T_{1}=0.29 \mathrm{eV}\right)$. Also for superphane 1 a small gap between the first and second triplet state is calculated $\left(T_{2}-T_{1}=0.26\right.$ $\mathrm{eV}$ ), but here only one triplet state could be observed. Obviously the necessary stabilization of the second triplet state by deformations is no 
longer possible for 1 due to the rigidity of the carbon skeleton.

\section{Appendix}

The probability density $\Gamma(1,2)$ of the two unpaired electrons in a triplet state with the normalization condition $\int \Gamma(1,2) \mathrm{d} \tau_{1} \mathrm{~d} \tau_{2}=1$ is inconvenient for a qualitative discussion since $\Gamma(1,2)$ represents a six-dimensional function. We can write $\Gamma(1,2)=[\Omega(1,2)]^{2} \quad$ where $\Omega(1,2)=2^{-1 / 2} \times$ $\sum_{(i, k)} C_{i k}\left[n_{i}(1) n_{k}(2)-n_{k}(1) n_{i}(2)\right]$ is an antisymmetric spatial function depending on the MOs $n_{i}=\Sigma_{s} c_{i s} X_{s}$ and the coefficients $C_{i k}$ of the singly excited configurations $\phi_{i \rightarrow k}$ in the configuration interaction wavefunction. By introducing the approximation $\int_{\mathrm{A}} X_{s}^{\mathrm{A}}(1) X_{t}^{\mathrm{B}}(1) \mathrm{d} \tau_{1}=\delta_{\mathrm{AB}} \delta_{s t}$ where the integration is confined to the space of subunit $A$ of the phane and $X_{s}^{\mathrm{A}}$ designates a basis function in A we arrive at the following expression for the probability $\Gamma_{\mathrm{AB}}$ for finding the second unpaired electron in $B$ if the first one is in $A$ :

$$
\begin{aligned}
\Gamma_{\mathrm{AB}}= & \left(2-\delta_{\mathrm{AB}}\right) \sum_{s \neq t}^{\mathrm{A} \mathrm{B}}\left[\frac{1}{2} \sum_{(i k)} C_{i k}^{2} f_{i k s t}^{2}\right. \\
& \left.+\sum_{(i k)<(l m)} C_{i k} C_{l m} f_{i k s t} f_{l m s t}\right] .
\end{aligned}
$$

Here $f_{i k s t}=c_{i s} c_{k t}-c_{k s} c_{i t}$. This probability possesses the correct normalization condition

$$
\sum_{\mathrm{A} \leqslant \mathrm{B}} \Gamma_{\mathrm{AB}}=1
$$

\section{References}

[1] H. Sternlicht and H.M. McConnel, J. Chem. Phys. 35 (1961) 1793.

[2] M. Schwoerer and H.C. Wolf, Mol. Cryst. 3 (1967) 177.

[3] R.M. Hochstrasser and T.S. Lin, J. Chem. Phys. 49 (1968) 4929.

[4] R.H. Clarke, R.E. Connors, H.A. Frank and J.C. Hoch, Chem. Phys. Letters 45 (1977) 523.

[5] D. Schweitzer, J.P. Colpa, J. Behnke, K.H. Hausser, M.W. Haenel and H.A. Staab, Chem. Phys. 11 (1975) 373.

[6] W. Goldacker, K.H. Hausser, D. Schweitzer and H.A. Staab, J. Luminescence 18/19 (1979) 415.

[7] D. Schweitzer, Habilitationsschrift, Universität Heidelberg, FRG (1980).

[8] R.P.H. Kooyman and T.J. Schaafsma, J. Mol. Struct. 60 (1980) 373

[9] F. Gückel, D. Schweitzer, J.P. Collman, S. Bencosme, E. Evitt and J. Sessler, Chem. Phys. 86 (1984) 161.

[10] H. Vogler, Mol. Phys. 47 (1982) 1179.

[11] C.J. Brown and A.G. Farthing, Nature 164 (1949) 915.

[12] G. Melzer, D. Schweitzer, K.H. Hausser, J.P. Colpa and M.W. Haenel, Chem. Phys. 39 (1979) 229.

[13] W. Goldacker, D. Schweitzer, K.P. Dinse and K.H Hausser, Chem. Phys. 48 (1980) 10.

[14] B. Kovac, M. Mohraz, E. Heilbronner, V. Boekelheide and H. Hopf, J. Am. Chem. Soc. 102 (1980) 4314.

[15] R. Gleiter, Tetrahedron Letters (1969) 4453.

[16] J. Spanget-Larsen, Theoret. Chim. Acta 64 (1983) 187.

[17] Y. Sekine, M. Brown and V. Boekelheide, J. Am. Chem. Soc. 101 (1979) 3126.

[18] P.F.T. Schirch and V. Boekelheide, J. Am. Chem. Soc. 101 (1979) 3125.

[19] R.G. Parr, Quantum theory of molecular electronic structure (Benjamin, New York, 1964).

[20] J. Zuclich, D. Schweitzer and H.A. Maki, Photochem. Photobiol. 18 (1973) 161.

[21] P.H. Scudder, V. Boekelheide, D. Cornutt and H. Hopf, Spectrochim. Acta 37a (1981) 425.

[22] H. Vogler, G. Ege and H.A. Staab, Mol. Phys. 33 (1977) 923.

[23] H. Hope, J. Bernstein and K.N. Truebbord, Acta Cryst. B 28 (1972) 1733

[24] A.W. Hanson and T.S. Comeron, J. Chem. Res. S (1980) 336.

[25] H. Vogler, unpublished results.

[26] S. Iwata and K.F. Freed, Chem. Phys. Letters 28 (1974) 176. 